\title{
Mass of the active galactic nucleus black hole XMMUJ134736.6+173403
}

\author{
K. Goluchová ${ }^{1}$, G. Török ${ }^{1}$, E. Šrámková1 ${ }^{1}$ M. A. Abramowicz ${ }^{1}$, Z. Stuchlík ${ }^{1}$, and J. Horák ${ }^{2}$ \\ 1 Institute of Physics, Research Centre for Computational Physics and Data Processing, Research Centre for Theoretical Physics and \\ Astrophysics, Faculty of Philosophy \& Science, Silesian University in Opava, Bezručovo nám. 13, 74601 Opava, Czech Republic \\ e-mail: katka.g@seznam.cz \\ 2 Astronomical Institute, Boční II 1401/2a, 14131 Praha 4-Spořilov, Czech Republic
}

Received 4 December 2018 / Accepted 9 January 2019

\begin{abstract}
A recent study of the X-ray source XMMUJ134736.6+173403 has revealed a strong quasi-periodic modulation in the X-ray flux. The observation of two quasiperiodic oscillations (QPOs) that occur on a daily timescale and exhibit a 3:1 frequency ratio strongly supports the evidence for the presence of an active galactic nucleus black hole (AGN BH). Assuming an orbital origin of QPOs, we calculated the upper and lower limit on AGN BH mass $M$ and found $M \approx 10^{7}-10^{9} M_{\odot}$. When we compare this to mass estimates of other sources, XMMUJ134736.6+173403 appears to be the most massive source with commensurable QPO frequencies, and its mass represents the current observational upper limit on AGN BH mass based on QPO observations. We note that it will be crucial for the falsification of particular resonance models of QPOs whether only a single QPO with a frequency that completes the harmonic sequence 3:2:1 is found in this source, or if a new different pair of QPOs with frequencies in the 3:2 ratio is found. The former case would agree with the prediction of the $3: 2$ epicyclic resonance model and $\mathrm{BH}$ mass $M \approx\left(5 a^{2}+8 a+8\right) \times 10^{7} M_{\odot}$, where $a$ is a dimensionless BH spin.
\end{abstract}

Key words. X-rays: binaries - black hole physics - accretion, accretion disks

\section{Introduction}

The X-ray power density spectra (PDS) of several Galactic black hole $(\mathrm{BH})$ systems displays so-called high-frequency quasiperiodic oscillations (HF QPOs) within the range of $40-450 \mathrm{~Hz}$. It has been noted that their frequencies lie within the range corresponding to timescales of orbital motion in the vicinity of a $\mathrm{BH}$. This coincidence is thought to represent a strong indication that the observed signal originates in the innermost parts of an accretion disk. This hypothesis also finds support in the field of Fourier-resolved spectroscopy (e.g., Gilfanov et al. 2000; McClintock \& Remillard 2006; van der Klis 2006).

Detections of elusive HF QPO peaks in Galactic microquasars are often reported at rather constant frequencies that are characteristic for a given source. It has been found that they usually appear in ratios of small natural numbers (Abramowicz \& Kluźniak 2001; Remillard et al. 2002; McClintock \& Remillard 2006), typically in a 3:2 ratio (see, however, Belloni et al. 2012; Belloni \& Altamirano 2013; Varniere \& Rodriguez 2018). The evidence for the rational frequency ratios was also discussed in the context of neutron star (NS) QPOs. In the NS sources, clustering of twin-peak QPO detections most frequently arises as a result of the weakness of (one or both) QPOs outside the limited range of QPO frequencies (frequency ratio; see Abramowicz et al. 2003; Belloni et al. 2005, 2007; Török et al. 2008a,b; Barret \& Boutelier 2008; Boutelier et al. 2010).

The 3:2 frequencies observed in the Galactic microquasars are well matched by the relation (McClintock \& Remillard
2006)

$v_{\mathrm{U}}=\frac{2.8 \mathrm{kHz}}{M^{*}}$,

where $v_{\mathrm{U}}$ is the higher of the two frequencies that form the $3: 2$ ratio, $R=v_{\mathrm{U}} / v_{\mathrm{L}}=3 / 2$, and the $\mathrm{BH}$ gravitational mass is given as $M^{*}=M / M_{\odot}$.

Abramowicz et al. (2004) suggested that detections of 3:2 QPOs and scaling Eq. (1) could provide the basis for a method intended to determine the mass of $\mathrm{BH}$ sources such as active galactic nuclei (AGN) and ultraluminuos X-ray (ULX) sources. It has been argued that a confirmation of 3:2 QPOs in other than stellar mass BH sources could be of fundamental importance for the BH accretion theory (Abramowicz et al. 2004; Török 2005a,b). It was furthermore discussed that within a wide range of BH masses $M$, both the rotational parameter $a \equiv \mathrm{c} J / \mathrm{GM}^{2}$ and specific details of a given orbital QPO model are of secondary importance, and that the observed frequencies can be used for to estimate $M^{1}$.

A full decade after these results have been published, Zhou et al. (2015) completed a survey pointing to a further observational evidence for the large-scale validity of the $1 / M$ scaling of the BH QPO frequencies (Eq. (1)). Even more important findings that support this evidence have been accumulated recently. At present, several sources that span the range of a few orders of magnitude seem to follow the relation. This is illustrated in Fig. 1, and further details along with several references can be found

In this paper we assume Kerr BH space times. 


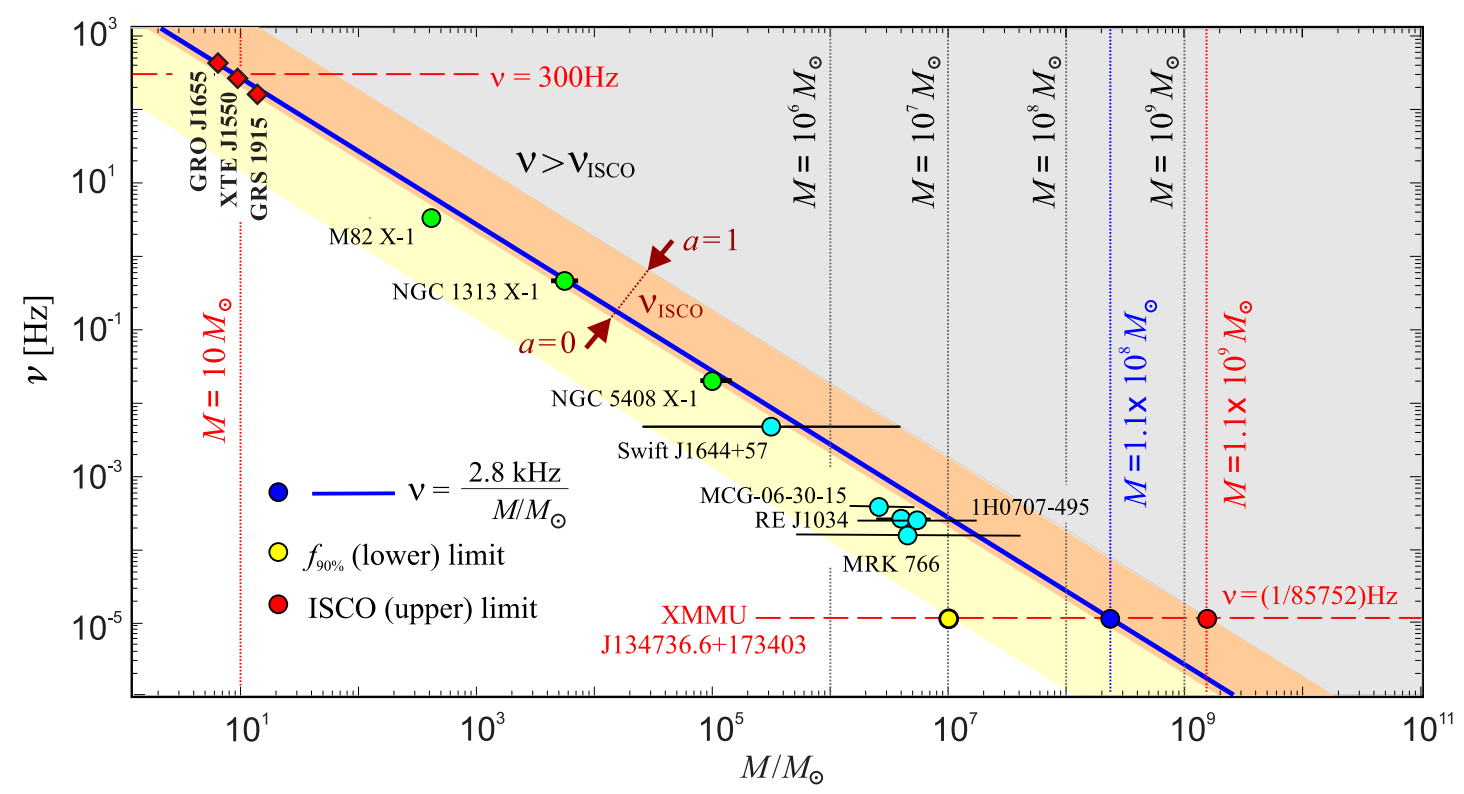

Fig. 1. Large scaling of BH HF QPO frequencies vs. Keplerian frequencies in the accretion disk. The upper left corner of the plot corresponds to Galactic microquasar BHs, while the lower right corner corresponds to supermassive BHs. The light orange region denotes the ISCO frequencies in the range of $a \in[0,1]$. The gray area indicates frequencies that are higher than the ISCO frequency. The light yellow area denotes Keplerian frequencies in the inner part of the (thin) disk that radiates more than $90 \%$ of the whole disk luminosity $(a \geq 0$, see Sect. 3). The green circles denote intermediate-mass BH sources whose mass estimate is based either fully or in a large part on the observations of HF QPOs. For some sources, the error bars are smaller than the displayed symbols.

in Zhou et al. (2015), Pasham et al. (2014, 2015), Czerny et al. (2016), Zhang et al. (2017, 2018), Gupta et al. (2018), and also Smith et al. (2018). We here discuss the implications of the latest QPO observations and mainly focus on the importance of QPOs that have recently been found in the X-ray source XMMUJ134736.6+173403 by Carpano \& Jin (2018).

\section{Upper bounds on AGN mass based on HF QPOs}

Carpano \& Jin (2018) carried out a systematic study of Chandra, Swift, and XMM-Newton observations of the X-ray source XMMUJ134736.6+173403, which has been previously found to be spatially coincident with a pair of galaxies including the Seyfert 2 galaxy SDSS J134736.39+173404.6. Using the Chandra observation from 2008, Carpano \& Jin (2018) accurately evaluated the position of this X-ray source and showed that its coordinates coincide with the position of the Seyfert 2 galaxy. Within the analysis of a set of 29 Swift observations conducted between February 6 and May 23, 2008, they discovered strong twin-peak quasi-periodic oscillations with periods of $23.82 \pm 0.07 \mathrm{~h}$ and $71.44 \pm 0.57 \mathrm{~h}$.

This measurement clearly indicates that $R=3.00 \pm$ 0.04 , and it provides very strong evidence for frequencycommensurable QPOs in an AGN BH. The frequency commensurability is crucial for the nonlinear resonance models discussed by Abramowicz, Kluzniak and collaborators (e.g., Abramowicz \& Kluźniak 2001; Rebusco 2004; Horák 2008; Török et al. 2005). Motivated by the observed 3:1 frequency ratio, Carpano \& Jin (2018) investigated the mass-spin relations expected for this source in detail from various resonances between disk-oscillation modes that have previously been discussed in the context of QPOs in Galactic microquasars. We make several remarks regarding these models in Sects. 3 and 4, where other QPO models are considered as well. In what follows, we explore general implications of the observed rapid variability that are valid for a large class of orbital QPO models.
The Keplerian frequency of matter orbiting a BH monotonically increases as the orbital radius $r$ decreases to the inner edge of the accretion disk. The location of the inner edge depends on the radiative efficiency of the disk. For a very high efficiency, the disk terminates at the marginally stable circular orbit (thin disks), $r_{\text {ISCO }}$ (often called innermost stable circular orbit, or ISCO), while for a very low efficiency, it terminates at the marginally bound orbit (ion tori, thin disks, and advection dominated accretion flows - ADAFs), $r_{\text {RIsco }}$. The Keplerian orbital frequency at these orbits for a Schwarzschild BH $(a=0)$ scales with BH mass as (e.g., Bardeen et al. 1972)

$v_{\mathrm{ISCO}}=\frac{2.20 \mathrm{kHz}}{M^{*}}, \quad v_{\mathrm{RISCO}}=\frac{4.04 \mathrm{kHz}}{M^{*}}$.

For rotating $\mathrm{BHs}$ (corrotating disks), these frequencies are higher, and for maximally rotating $\operatorname{Kerr} \mathrm{BH}(a=1)$, we may write

$v_{\mathrm{ISCO}}=v_{\mathrm{RISCO}}=\frac{16.2 \mathrm{kHz}}{M^{*}}$.

In Fig. 1 we present relations that determine the highest orbital frequencies. We also mark here the higher QPO frequency observed in XMMUJ134736.6+173403. Postulating that this frequency corresponds to the Keplerian frequency inside the disk, we can find that the mass of the source should not be higher than $M \doteq 1.1 \times 10^{9} M_{\odot}$.

\section{Application of particular orbital models of QPOs and lower bounds on AGN mass}

A large group of models, to which we refer as standard geodesic orbital (SGO) models, assume that the observed frequencies, $v_{\mathrm{L}}$ and $v_{\mathrm{U}}$, are equal to fundamental frequencies of (geodesic) orbital motion, that is, the Keplerian frequency defined at a circular orbit above ISCO, $r \geq r_{\text {ISco }}$, and the radial and vertical 
a)

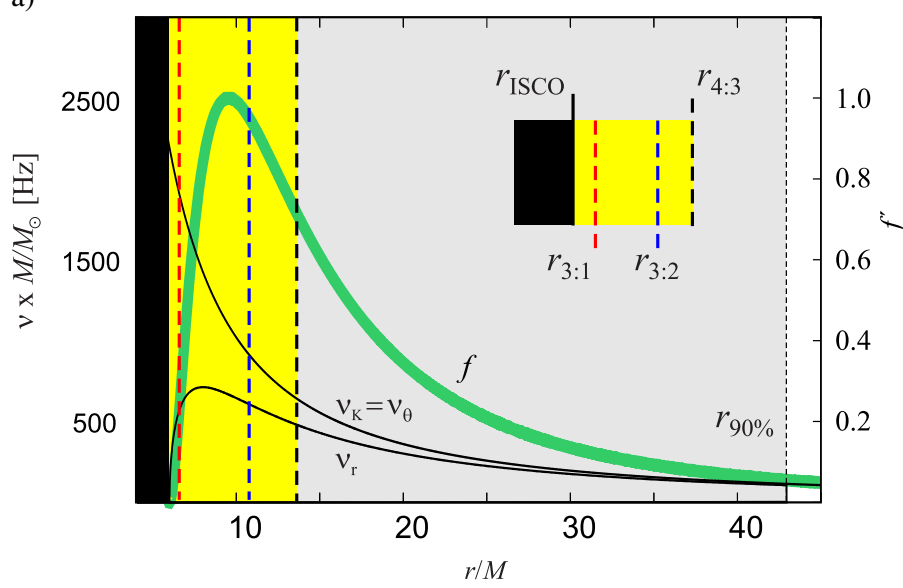

b)

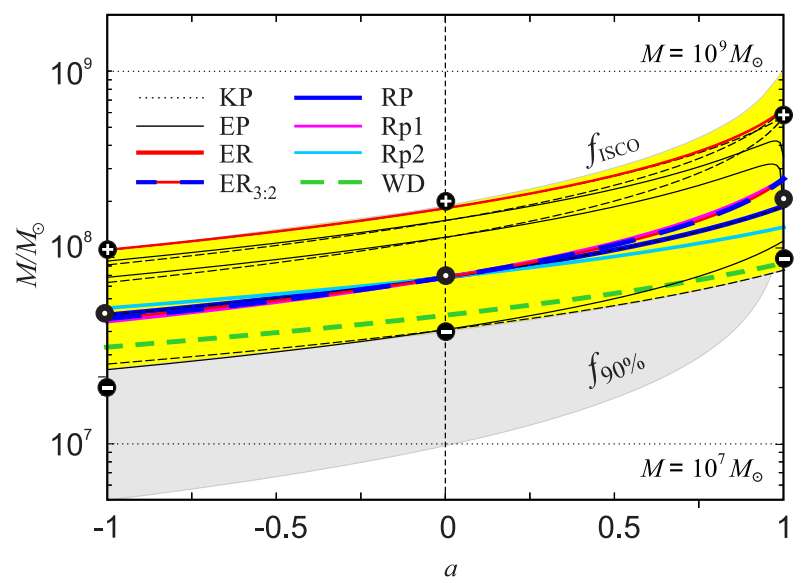

Fig. 2. Panel $a$ : radial profiles of the normalized disk flux (thin relativistic disk) and the Keplerian and epicyclic frequencies of geodesic orbital motion calculated for a Schwarzschild $\mathrm{BH}$. The gray region between $r_{\text {ISco }}$ and $r_{90 \%}$ emphasizes the radial part of the disk that radiates more than $90 \%$ of the whole disk luminosity. Several particular resonant orbits are denoted. Indexes $i$ and $j$ in $r_{i: j}$ are chosen such that $i / j=v_{\theta} / v_{\mathrm{r}}$. Panel $b$ : mass-spin relations inferred for XMMUJ134736.6+173403 from the observed QPO frequencies and their models in Kerr spacetimes. The solid and dashed black lines are drawn for the set of resonance models considered by Carpano \& Jin (2018). The red line denotes the prediction of the ER model, while the blue line corresponds to the RP model. The dashed red-blue line denotes the consideration of the $E R_{3: 2}$ model. The gray region corresponds to the association of the QPO frequency with the Keplerian frequency at orbits inside a part of the disk that radiates more than $90 \%$ of the whole disk luminosity. The lower boundary of the gray region corresponds to $r_{90 \%}$, while the upper boundary corresponds to $r_{\text {ISCo }}$. The yellow region emphasizes the range covered by the assumed QPO models. The nine spots denote boundaries of relation (5) for $a \in[-1,1]$.

epicyclic frequency, or to their linear combinations (including the periastron and Lense-Thirring precession frequency). The three frequencies $v_{\mathrm{K}}, v_{\mathrm{r}}$, and $v_{\theta}$ can be written in a general form

$v_{i}=\frac{1}{T_{i}}=\frac{c^{3}}{2 \pi G} \frac{1}{M} \mathcal{F}_{i}(x, a) \leq v_{\mathrm{ISCO}}(M, a)$,

where $x=r / r_{\mathrm{G}} \geq r_{\mathrm{ISCO}} / r_{\mathrm{G}}, r_{\mathrm{G}}=G M / c^{2}$, and $\mathcal{F}_{i}$ is a specific function that for a fixed $\mathrm{BH}$ spin depends only on $x$. The $1 / M$ term here provides a physical explanation of the empiric scaling Eq. (1). We illustrate the behavior of Keplerian and epicyclic frequencies for the simple case of a Schwarzschild BH $(a=0)$ in Fig. 2a. We also include in this figure an illustration of the behavior of the normalized disk flux $f=f / f_{\max }$ calculated for relativistic thin disks (Page \& Thorne 1974).

The family of SGO models includes various physical concepts that hold the assumption that the QPO excitation radii are located within the most luminuos accretion region $(r \ll$ $r_{90 \%}$, see Fig. 2a), usually below $20 r_{\mathrm{G}}$. Several models assume, for instance, that QPOs are produced by a local motion of accreted inhomogeneities such as blobs or vortices. This subset of SGO models includes the so-called relativistic precession model (RP) or tidal disruption (TD) model (Abramowicz et al. 1992; Stella \& Vietri 1998; Stella et al. 1999; Čadež et al. 2008; Kostić et al. 2009; Bakala et al. 2014; Karssen et al. 2017; Germanà 2017). Another possibility is to relate the QPOs to a collective motion of the accreted matter, in particular, to some accretion disk oscillatory modes (Wagoner et al. 2001; Rezzolla et al. 2003; Abramowicz et al. 2006; Ingram \& Done 2010; Fragile et al. 2016). Specific models of this type often deal with oscillations in a slender accretion torus and assume some kind of resonance between these oscillation modes.

A general idea considering such resonances has originated in Abramowicz \& Kluźniak (2001; see also Aliev \& Galtsov 1981) and was later continued in the context of BH QPOs and spin extensively discussed in several subsequent works covering both parametric and forced resonances (Török et al. 2005, 2011;
Török 2005a,b; Stuchlík et al. 2013). Various combinations of both axisymmetric and non-axisymmetric epicyclic modes (EP class of models), as well as combinations of these modes and the Keplerian oscillation (KP class of models), have been considered. An often-quoted model is represented by the 3:2 epicyclic resonance model, or by a similar model that consideres the Keplerian frequency instead of the vertical epicyclic frequency (ER and KeP models).

The mass-spin relations predicted for Galactic microquasars by several QPO models have been investigated in a series of works (Török et al. 2011; Kotrlová et al. 2014, 2017). Along with the TD, RP, ER, and KeP model, these include the specific diskoseismic model of Kato $(2001,2007,2008)$ and two models introduced by Bursa et al. (2005) and Török et al. (2007, 2010) that were motivated by the observations of NS QPOs (the RP1 and RP2 models). We recall in Table1 how the QPO frequencies are identified with orbital frequencies of geodesic motion.

We calculated the mass-spin relations for XMMUJ134736.6+173403 based on the models listed in Table 1. The obtained results are presented in Fig. $2 \mathrm{~b}$ along with all mass-spin relations relevant to the models considered by Carpano \& Jin (2018). The mass predicted by these models is always below the ISCO limit $M \doteq 1.1 \times 10^{9} M_{\odot}$. It is nevertheless close to this limit (see the horizontal range of Fig. 1), and there is $10^{7} M_{\odot}<M<10^{9} M_{\odot}$, which is generic for the proposed SGO models when $a \geq 0$ because they involve QPO excitation radii $r<r_{90 \%}$. In Fig. $2 \mathrm{~b}$ we illustrate these findings and plot functions $f_{90 \%} \equiv M\left(r_{90 \%}\right)$ and $f_{\text {ISCO }} \equiv M\left(r_{\text {ISCO }}\right)$ that correspond to an identification of the observed QPO frequency with the appropriate Keplerian frequency.

\section{Implications for models assuming parametric resonances}

Kluźniak \& Abramowicz (2002) have suggested that the lower and upper $\mathrm{kHz}$ QPOs may be identified with the radial and vertical axisymmetric epicyclic oscillations of an accretion disk. 
Table 1. Frequency relations corresponding to individual QPO models investigated in Török et al. (2011).

\begin{tabular}{lll}
\hline \hline Model & \multicolumn{2}{c}{ Relations } \\
\hline $\mathrm{RP}$ & $v_{\mathrm{L}}=v_{\mathrm{K}}-v_{\mathrm{r}}$ & $v_{\mathrm{U}}=v_{\mathrm{K}}$ \\
$\mathrm{TD}^{a}$ & $v_{\mathrm{L}}=v_{\mathrm{K}}$ & $v_{\mathrm{U}}=v_{\mathrm{K}}+v_{\mathrm{r}}$ \\
\hline $\mathrm{WD}$ & $v_{\mathrm{L}}=2\left(v_{\mathrm{K}}-v_{\mathrm{r}}\right)$ & $v_{\mathrm{U}}=2 v_{\mathrm{K}}-v_{\mathrm{r}}$ \\
$\mathrm{ER}^{b}$ & $v_{\mathrm{L}}=v_{\mathrm{r}}$ & $v_{\mathrm{U}}=v_{\theta}$ \\
$\mathrm{KeP}^{b}$ & $v_{\mathrm{L}}=v_{\mathrm{r}}$ & $v_{\mathrm{U}}=v_{\mathrm{K}}$ \\
$\mathrm{RP} 1$ & $v_{\mathrm{L}}=v_{\mathrm{K}}-v_{\mathrm{r}}$ & $v_{\mathrm{U}}=v_{\theta}$ \\
$\mathrm{RP} 2$ & $v_{\mathrm{L}}=v_{\mathrm{K}}-v_{\mathrm{r}}$ & $v_{\mathrm{U}}=2 v_{\mathrm{K}}-v_{\theta}$ \\
\hline
\end{tabular}

Notes. The relations are expressed in terms of three fundamental frequencies of perturbed circular geodesic motion. ${ }^{(a)}$ Model is not consistent with the observed $3: 1$ frequency ratio. ${ }^{(b)}$ Model overlaps with the consideration of Carpano \& Jin (2018).

In their scenario, the resonance arises as a result of nonlinear coupling between the oscillation modes. The radial mode supplies energy to the vertical mode by means of the parametric resonance. As the parametric resonance occurs when it is $v_{\mathrm{r}} / v_{\theta}=2 / n$ (with $n$ being an integer number) and $v_{\mathrm{r}} \leq v_{\theta}$, their model naturally explains the $3: 2$ frequency ratio that is often observed in the QPO sources. A quantitative analysis of this model has been presented by Rebusco (2004) and Horák (2008). The latter work is devoted to a detailed study of the nature of nonlinear coupling between disk oscillation modes and extends the previous analysis to non-axisymmetric epicyclic modes. Consideration of these modes implies frequency identifications of the lower and upper QPO that involve combinations of both Keplerian and epicyclic frequencies because they oscillate with frequencies $m v_{\mathrm{K}} \pm v_{\mathrm{r}, \theta}$, where $m$ is an integer azimuthal wavenumber.

\subsection{Restrictions on resonant interactions}

Formulae of the Ep, RP1, and RP2 models from Table 1 combine various combinations of the radial and vertical epicylic modes with azimuthal wavenumbers $\left(m_{\mathrm{r}}, m_{\theta}\right)=(0,0),(1,0)$, and $(1,2)$, respectively. The symmetry properties of the accretion flow strongly limit the resonant interaction between the modes. In particular, when the unperturbed flow is both axially symmetric and symmetric with respect to the equatorial plane, possible resonances up to the fourth order occur only when $v_{\theta} / v_{r}=1 / 2,1,1 / 4$, and $3 / 2$, and the same condition has also to be satisfied by the azimuthal wavenumbers, that is, $m_{\theta} / m_{\mathrm{r}}=1 / 2,1,1 / 4$ or $3 / 2$. In this context, in the case of the 3:2 observed QPO frequency ratio, the idea of parametric resonance is consistent only with the frequency identification provided by the ER model (Horák 2008). Even this model nevertheless fails in explaining the 3:1 frequency ratio observed in XMMUJ134736.6+173403.

\section{2. $3: 2$ resonance}

A resolution of the problem may be connected to a nonlinear nature of both the flow oscillations and the modulation mechanism through which the oscillations manifest themselves in the observed light curves. The observed power spectra often contain higher harmonics and subharmonics of the fundamental mode frequencies. The 3:1 frequency ratio then still may be explained by the 3:2 resonance provided that the first-order harmonics of the upper fundamental mode, or a subharmonic of the lower fun- damental mode, is observed. We illustrate the underlying massspin relation in Fig. 2. It is considered that $v_{\mathrm{U}}$ equals $v_{\theta}$ and $v_{\mathrm{L}}$ equals $v_{\theta}-v_{\mathrm{r}}\left(E R_{3: 2}\right.$ model $)$.

\section{Discussion and conclusions}

If the observed BH HF QPOs can be described by a model similar to some of the SGO models, XMMUJ134736.6+173403 likely represents the most massive $\mathrm{BH}$ source with commensurable QPO frequencies. Based on the mass estimation implied by the ISCO limit, we can establish $M \approx 10^{9} M_{\odot}$ as the current observational upper limit on an AGN BH mass inferred from QPOs. This limit is valid as long as the real oscillatory frequencies of the disk are not much higher than the Keplerian frequency. This case is nevertheless not very likely to occur (e.g., Straub \& Šrámková 2009). We note that consideration of miscellaneous combinational frequencies within the QPO models may lead to obtaining observable frequencies that are (although not much) higher than the Keplerian limit.

\subsection{Local versus global models, resonances}

The problem of determination of the right qpo model exceeds the scope of this Letter. It is nevertheless worthwhile to note that HF QPOs commonly found in Seyefert 1 and Seyefert 2 AGNs pose a challenge for physical concepts that have been proposed to explain modulation of the observed flux within the QPO models.

Seyfert 1 AGNs are expected to have accretion disks with small inclination angles (i.e., disks that are nearly parallel to the observer's sky). Models that include orbiting accreted inhomogeneities assume a rather small extent of the modulated region of the disk, which implies a low ratio of the modulated to total disk flux. This ratio often approaches zero for the nearly face-on view (e.g, Schnittman \& Bertschinger 2004). Either somewhat non-equatorial orbits or a very powerful enhancement of the inhomogeneities are therefore required in order to explain the observed source variability (e.g., tidal disruption events). In principle, models that introduce a collective motion of the accreted fluid allow for a high spatial extent of the modulated accretion region (e.g., Wagoner et al. 2001; Bursa et al. 2004; Schnittman \& Rezzolla 2006; Ingram \& Done 2010; Bakala et al. 2014). In this sense, this group of models that include the diskoseismic models and concepts that incorporate oscillating tori or hot inner accretion flow are favored.

Regarding the latter class of models, we suggest that it will be crucial for the falsification of models that are based on parametric resonances between disk oscillation modes whether only single QPO with a frequency that completes the harmonic sequence 3:2:1 is found in this source, or if a new different pair of QPOs with frequencies in the 3:2 ratio is found. The former case would agree with the prediction of the 3:2 epicyclic resonance $\left(E R_{3: 2}\right)$ model, which explains the observed 3:1 frequency ratio by means of the combinational frequencies. For both corotating and counterrotating disks, the corresponding mass-spin relation shown in Fig. 2 (the dashed red-blue line) can be matched by a simple approximative quadratic relation, $M \approx\left(5 a^{2}+8 a+8\right) \times 10^{7} M_{\odot}$.

\subsection{Quantification of results common to SGO models}

Figure 2b shows that the $E R_{3: 2}$ model prediction coincides for $a=0$ with the predictions of several other models such as the RP 
model. On a large scale of $M$, all the considered models provide similar mass-spin relations. Following the quadratic approximation, we may write

$M \approx\left(5_{-3}^{+11} a^{2}+8_{-4}^{+17} a+8_{-4}^{+12}\right) \times 10^{7} M_{\odot}$,

where the upper limit corresponds to models that require a high BH mass (e.g., the ER model) and the lower limit corresponds to models that require a low BH mass (e.g., the WD model). The boundaries of relation Eq. (5) for $a \in[-1,1]$ are denoted by spots in Fig. 2b. This figure shows that relation Eq. (5) describes the predictions of the whole group of the considered SGO models well.

Acknowledgements. We would like to acknowledge the Czech Science Foundation grant No. 17-16287S, the INTER-EXCELLENCE project No. LTI17018 that supports the collaboration between the Silesian University in Opava and the Astronomical Institute in Prague, and internal grants of the Silesian University, SGS/14,15/2016. We thank the anonymous referee for their comments and suggestions that have greatly helped us to improve the paper.

\section{References}

Abramowicz, M. A., \& Kluźniak, W. 2001, A\&A, 374, L19

Abramowicz, M. A., Lanza, A., Spiegel, E. A., \& Szuszkiewicz, E. 1992, Nature, 356,41

Abramowicz, M. A., Almergren, G. J. E., Kluźniak, W., \& Thampan, A. V. 2003, ArXiv e-prints [arXiv:gr-qc/0312070]

Abramowicz, M. A., Kluźniak, W., McClintock, J. E., \& Remillard, R. A. 2004, ApJ, 609, L63

Abramowicz, M. A., Blaes, O. M., Horák, J., Kluźniak, W., \& Rebusco, P. 2006, Class. Quant. Grav., 23, 1689

Aliev, A. N., \& Galtsov, D. V. 1981, Gen. Relativ. Gravitation, 13, 899

Bakala, P., Török, G., Karas, V., et al. 2014, MNRAS, 439, 1933

Bardeen, J. M., Press, W. H., \& Teukolsky, S. A. 1972, ApJ, 178, 347

Barret, D., \& Boutelier, M. 2008, New Astron. Rev., 51, 835

Belloni, T. M., \& Altamirano, D. 2013, MNRAS, 432, 10

Belloni, T., Méndez, M., \& Homan, J. 2005, A\&A, 437, 209

Belloni, T., Homan, J., Motta, S., Ratti, E., \& Méndez, M. 2007, MNRAS, 379, 247

Belloni, T. M., Sanna, A., \& Méndez, M. 2012, MNRAS, 426, 1701

Boutelier, M., Barret, D., Lin, Y., \& Török, G. 2010, MNRAS, 401, 1290

Bursa, M. 2005, in RAGtime 6/7: Workshops on black holes and neutron stars, eds. S. Hledík, \& Z. Stuchlík, 39

Bursa, M., Abramowicz, M. A., Karas, V., \& Kluźniak, W. 2004, ApJ, 617, L45

Carpano, S., \& Jin, C. 2018, MNRAS, 477, 3178

Czerny, B., You, B., Kurcz, A., et al. 2016, A\&A, 594, A102
Fragile, P. C., Straub, O., \& Blaes, O. 2016, MNRAS, 461, 1356 Germanà, C. 2017, PRD, 96, 103015

Gilfanov, M., Churazov, E., \& Revnivtsev, M. 2000, MNRAS, 316, 923

Gupta, A. C., Tripathi, A., Wiita, P. J., et al. 2018, A\&A, 616, L6

Horák, J. 2008, A\&A, 486, 1

Ingram, A., \& Done, C. 2010, MNRAS, 405, 2447

Karssen, G. D., Bursa, M., Eckart, A., et al. 2017, MNRAS, 472, 4422

Kato, S. 2001, PASJ, 53, 1

Kato, S. 2007, PASJ, 59, 451

Kato, S. 2008, PASJ, 60, 111

Kluźniak, W., \& Abramowicz, M. A. 2002, ArXiv e-prints

[arXiv:astro-ph/0203314]

Kostić, U., Čadež, A., Calvani, M., \& Gomboc, A. 2009, A\&A, 496, 307

Kotrlová, A., Török, G., Šrámková, E., \& Stuchlík, Z. 2014, A\&A, 572, A79

Kotrlová, A., Šrámková, E., Török, G., Stuchlík, Z., \& Goluchová, K. 2017, A\&A, 607, A69

McClintock, J. E., \& Remillard, R. A. 2006, Black hole binaries (Cambridge: Cambridge University Press), 157

Page, D. N., \& Thorne, K. S. 1974, ApJ, 191, 499

Pasham, D. R., Strohmayer, T. E., \& Mushotzky, R. F. 2014, Nature, 513, 74

Pasham, D. R., Cenko, S. B., Zoghbi, A., et al. 2015, ApJ, 811, L11

Rebusco, P. 2004, PASJ, 56, 553

Remillard, R. A., Muno, M. P., McClintock, J. E., \& Orosz, J. A. 2002, ApJ, 580, 1030

Rezzolla, L., Yoshida, S., \& Zanotti, O. 2003, MNRAS, 344, 978

Schnittman, J. D., \& Bertschinger, E. 2004, ApJ, 606, 1098

Schnittman, J. D., \& Rezzolla, L. 2006, ApJ, 637, L113

Smith, K. L., Mushotzky, R. F., Boyd, P. T., \& Wagoner, R. V. 2018, ApJ, 860, L10

Stella, L., \& Vietri, M. 1998, AJ, 492, L59

Stella, L., Vietri, M., \& Morsink, S. M. 1999, AJ, 524, L63

Straub, O., \& Šrámková, E. 2009, Class. Quant. Grav., 26, 055011

Stuchlík, Z., Kotrlová, A., \& Török, G. 2013, A\&A, 552, A10

Török, G. 2005a, ApJ, 440,

Török, G. 2005b, Astron. Nachr., 326, 856

Török, G., Abramowicz, M. A., Kluźniak, W., \& Stuchlík, Z. 2005, A\&A, 436,

Török, G., Stuchlík, Z., \& Bakala, P. 2007, Cent. Eur. J. Phys., 5, 457

Török, G., Abramowicz, M. A., Bakala, P., et al. 2008a, Acta Astron., 58, 15

Török, G., Bakala, P., Stuchlik, Z., \& Čech, P. 2008b, Acta Astron., 58, 1

Török, G., Bakala, P., Šrámková, E., Stuchlík, Z., \& Urbanec, M. 2010, AJ, 714, 748

Török, G., Kotrlová, A., Šrámková, E., \& Stuchlík, Z. 2011, A\&A, 531, A59

Čadež, A., Calvani, M., \& Kostić, U. 2008, A\&A, 487, 527

van der Klis, M. 2006, Rapid X-ray Variability (Cambridge: Cambridge University Press), 39

Varniere, P., \& Rodriguez, J. 2018, ApJ, 865, 113

Wagoner, R. V., Silbergleit, A. S., \& Ortega-Rodríguez, M. 2001, AJ, 559, L25

Zhang, P., Zhang, P.-F., Yan, J.-Z., Fan, Y.-Z., \& Liu, Q.-Z. 2017, ApJ, 849, 9

Zhang, P.-F., Zhang, P., Liao, N.-H., et al. 2018, ApJ, 853, 193

Zhou, X.-L., Yuan, W., Pan, H.-W., \& Liu, Z. 2015, ApJ, 798, L5 